\title{
Evidence for Clinicians
}

\section{Routine oro/nasopharyngeal suction versus no suction at birth}

\author{
Manoj Kumar MBBS MD MSc (Clinical Epidemiology) \\ Neonatal Division, Department of Pediatrics, University of Alberta, Edmonton, Alberta \\ Correspondence: Manoj Kumar, Neonatal Division, Department of Pediatrics, University of Alberta, Edmonton, Alberta. \\ Telephone +1 780-735-4670, fax: +1 780-735-4072, E-mail: manojk@ualberta.ca
}

For the current issue of the Journal, we asked Dr. Manoj Kumar to comment on and put into context the recent Cochrane Review on routine nasal suction at birth.

\section{BACKGROUND}

Oro/nasopharyngeal suction is a method used to clear secretions from the oropharynx and nasopharynx through the application of negative pressure via a suction catheter or bulb syringe. Traditionally, airway oro/nasopharyngeal suction at birth has been used routinely to remove fluid rapidly from the oropharynx and nasopharynx in vigorous and nonvigorous infants at birth. Concerns relating to the reported adverse effects of oro/nasopharyngeal suctioning led to a practice review and routine oro/ nasopharyngeal suctioning is no longer recommended for vigorous infants. However, it is important to know whether there is any clear benefit or harm for infants whose oro/nasopharyngeal airway is suctioned compared with infants who are not suctioned.

\section{OBJECTIVES}

The objective of this study was to evaluate the effect of routine oropharyngeal/nasopharyngeal suction compared with no suction on mortality and morbidity in newly born infants.

\section{SEARCH METHODS}

We used the standard search strategy of the Cochrane Neonatal Review group to search the Cochrane Central Register of Controlled Trials (CENTRAL 2016, Issue 3), MEDLINE via PubMed (1966 to April 18, 2016), Embase (1980 to April 18, 2016), and CINAHL (1982 to April 18, 2016). We also searched clinical trials databases, conference proceedings, and the reference lists of retrieved articles for randomized controlled trials and quasi-randomixed trials.

\section{SELECTION CRITERIA}

Randomized, quasi-randomized controlled trials and cluster randomized trials that evaluated the effect of routine oropharyngeal/nasopharyngeal suction compared to no suction on mortality and morbidity in newly born infants with and without meconium-stained amniotic fluid (MSAF).

\section{DATA COLLECTION AND ANALYSIS}

The review authors extracted from the reports of the clinical trials, data regarding clinical outcomes including mortality, need for resuscitation, admission to neonatal intensive care, 5-minute Apgar score, episodes of apnoea, and length of hospital stay.

\section{Main Results}

Eight randomized controlled trials met the inclusion criteria and only included term infants $(n=4,011)$. Five studies included infants with no fetal distress and clear amniotic fluid, one large study included vigorous infants with clear or MSAF, and two large studies included infants with thin or thick MSAF. Overall, there was no statistical difference between oro/nasopharyngeal suction and no oro/nasopharyngeal suction for all reported outcomes: mortality (typical RR 2.29, $95 \%$ confidence interval [CI] 0.94 to 5.53 ; typical $\mathrm{RD}$ $0.01,95 \% \mathrm{CI}-0.00$ to $0.01 ; \mathrm{I} 2=0 \%$, studies $=2$, participants $=3,023$ ), need for resuscitation (typical RR0.85, 95\% CI 0.69 to 1.06 ; typical $\mathrm{RD}-0.01,95 \% \mathrm{CI}-0.03$ to 0.00 ; $\mathrm{I} 2=$ $0 \%$, studies $=5$, participants $=3,791)$, admission to neonatal intensive care unit (typical RR 0.82, 95\% CI 0.62 to 1.08 ; typical RD $-0.03,95 \%$ CI -0.08 to 0.01 ; I2 = 27\%, studies $=2$, participants $=997)$ and Apgar scores at 5 minutes ( $\mathrm{MD}-0.03,95 \% \mathrm{CI}-0.08$ to 0.02 ; I 2 not estimated, studies $=3$, participants $=330)$. 


\section{AUTHORS' CONCLUSIONS}

The currently available evidence does not support or refute the benefits or harms of routine oro/nasopharyngeal suction over no suction. Further high-quality studies are required in preterm infants or term newborn

The full text of the Cochrane Review is available in The Cochrane Library: Foster JP, Dawson JA, Davis PG, Dahlen HG. Routine oro/nasopharyngeal suction versus no suction at birth. Cochrane Database Syst Rev 2017, Issue 4. Art. No.: CD010332. doi:10.1002/14651858.CD010332.pub2

\section{EXPERT COMMENTARY}

The Neonatal Resuscitation Program (NRP) guidelines have significantly changed over time in terms of the recommendations on oro/nasopharyngeal suctioning in neonates following birth. The earlier versions of the guidelines had suggested routine oro/nasopharyngeal suctioning for all newborns to establish effective ventilation and to facilitate their transition to extrauterine life (1). However, oro/nasopharyngeal suctioning, carried out in settings of clear amniotic fluid, was noted to result in more bradycardia episodes in infants and lower oxygen saturation without any demonstrable benefits $(2,3)$. The current NRP guidelines (4) recommend suctioning of oro/nasopharynx after birth in the following clinical settings: 1) if the baby is not breathing, is gasping, has poor tone, 2) if secretions appear to be obstructing the airway, 3 ) if the baby is having difficulty clearing their secretions, 4) if there is MSAF, or 5) if anticipating starting positive pressure ventilation (PPV). Most centers in Canada regularly train their neonatal health care providers in the most recent NRP guidelines and strive to adhere to these guidelines in practice.

The results of this Cochrane review (5) evaluating the effect of routine oro/nasopharyngeal suctioning following delivery, as compared with no suctioning, in settings of clear or MSAF, do not support or refute the benefits or harm of this practice. The authors indicate that the data from existing trials are insufficient to guide the practice of oro/nasopharyngeal suctioning in a decisive manner, and that providers could choose to do oro/ nasopharyngeal suctioning or skip it as part of initial steps of resuscitation of newborn. Being inconclusive, these results are neither an endorsement nor a rebuke of the practice of oro/nasopharyngeal suctioning in specific clinical situations as mentioned in the current NRP guidelines.

There is a general agreement among the neonatal community that the routine oro/nasopharyngeal suctioning of all infants born in settings of clear amniotic fluids is not warranted. Oro/nasopharyngeal suctioning of a vigorous infant is usually avoided in the case rooms these days and this approach is likely to continue with the publication of this review. However, withholding oro/nasopharyngeal suctioning, from infants that are unable to clear their secretions or are not vigorous and require initiation of PPV, is unlikely to be accepted in clinical practice on the basis of this review, as a general principle of establishing effective breathing in a critical patient, is to rapidly clear airways from secretions before initiating assisted ventilation. Thus, irrespective of the inconclusive results from this review, oro/nasopharyngeal suctioning is likely to continue in these clinical situations.

For births in settings of MSAF, oro/nasopharyngeal suctioning is currently only carried out after the infant is brought to the resuscitation table. Not so long ago, such infants were also suctioned at the perineum following the delivery of head, before they could take their first breath. However, Vain et al. (6), in a large RCT published in 2004, showed the futility of oro/nasopharyngeal suctioning at perineum in such cases, irrespective of the consistency of the meconium. As such, in the last decade or so, the practice of intrapartum suctioning of infant at perineum following the delivery of head has been nearly abandoned in the case rooms in Canada. This Cochrane review confirms the lack of benefit from this practice.

The review did not find any evidence in support of the practice of oro/nasopharyngeal suctioning in presence of MSAF, after the infant has initiated breathing efforts. However, the practice was evaluated only in a small subset of patients in one of the trials included in the Cochrane review, where investigators randomized subjects to oro/nasopharyngeal suctioning by a bulb syringe versus gentle wiping of the face and mouth with a towel (7). Although, the current NRP guidelines suggest oro/ nasopharyngeal suctioning in such cases, my experience has been that it is infrequently carried out if the infant is noted to be vigorous. As such, the initial steps of resuscitation of infants born in the settings of MSAF have become fairly similar to those born in presence of clear amniotic fluid, and are determined by the tone and the breathing effort of the infant. The practice is likely to be unaffected from the results of this Cochrane review.

In conclusion, the results of this Cochrane review are equivocal in terms of whether there are any benefits from routine oro/ nasopharyngeal suctioning of infants at birth. However, the often cited adage in medicine, that is, 'absence of evidence is not evidence of absence' (8), seems particularly to be the case here and is a significant limitation of the results of this review in terms of applicability for clinical practice. The review contains sparse data on clinical situations where oro/nasopharyngeal suctioning is currently being practiced as per the current NRP guidelines (4). Another significant limitation of the evidence from this review is that none of the included trials enrolled preterm infants; as such the role of oro/nasopharyngeal suctioning in this specific population remains unknown. Preterm infants may not able to clear their oro/nasopharyngeal secretions as effectively as term infants and thus may need help with suctioning. 
Also, preterm infants are more likely to need PPV at birth and thus are more likely to need suctioning to clear oropharyngeal secretions. While, the results of this review suggest lack of evidence for oro/nasopharyngeal suctioning in infants following delivery, they are unlikely to impact the current clinical practice in North America due to the limitations stated above. Further well-designed clinical trials, evaluating the practice of oro/nasopharyngeal suctioning in hypotonic and very preterm infants who may otherwise be unable to clear their oropharyngeal secretions effectively in the delivery room, are warranted. These trials should be adequately powered for meaningful clinical outcomes. In my practice, I generally avoid using oro/nasopharyngeal suctioning in any vigorous infant but will continue to use suctioning for indications suggested by the current NRP guidelines, particularly so for infants having difficulty clearing secretions or who are nonvigorous prior to initiating PPV.

Funding information: There are no funders to report for this submission. Potential Conflicts of Interest: No reported conflicts of interest. All authors have submitted the ICMJE Form for Disclosure of Potential Conflicts of
Interest. Conflicts that the editors consider relevant to the content of the manuscript have been disclosed.

\section{References}

1. American Heart Association; American Academy of Pediatrics. 2005 American Heart Association (AHA) guidelines for cardiopulmonary resuscitation (CPR) and emergency cardiovascular care (ECC) of pediatric and neonatal patients: Neonatal resuscitation guidelines. Pediatrics 2006;117(5):e1029-38.

2. Carrasco M, Martell M, Estol PC. Oronasopharyngeal suction at birth: Effects on arterial oxygen saturation. J Pediatr 1997;130(5):832-4.

3. Gungor S, Teksoz E, Ceyhan T, Kurt E, Goktolga U, Baser I. Oronasopharyngeal suction versus no suction in normal, term and vaginally born infants: A prospective randomised controlled trial. Aust N Z J Obstet Gynaecol 2005;45(5):453-6.

4. Initial Steps of Newborn Care. In: Weiner GM, Zaichkin JG, eds. Textbook of Neonatal Resuscitation (NRP), 7th edn. Elk Grove Village: American Academy of Pediatrics; 2016. ISBN (paper): 978-1-61002-024-4

5. Foster JP, Dawson JA, Davis PG, Dahlen HG. Routine oro/nasopharyngeal suction versus no suction at birth. Cochrane Database Syst Rev 2017;4:CD010332.

6. Vain NE, Szyld EG, Prudent LM, Wiswell TE, Aguilar AM, Vivas NI. Oropharyngeal and nasopharyngeal suctioning of meconium-stained neonates before delivery of their shoulders: Multicentre, randomised controlled trial. Lancet 2004;364(9434):597-602.

7. Kelleher J, Bhat R, Salas AA, et al. Oronasopharyngeal suction versus wiping of the mouth and nose at birth: A randomised equivalency trial. Lancet 2013;382(9889):326-30.

8. Altman DG, Bland JM. Absence of evidence is not evidence of absence. BMJ $1995 ; 311(7003): 485$. 